\title{
Dark Neurons: A protective mechanism or a mode of death
}

\section{Review Article}

\author{
Shahriar Ahmadpour', Arman Behrad², Iván Fernández-Vega3 \\ 1,2Anatomy Department, Medicine Faculty, North Khorasan University of Medical Sciences, \\ Bojnurd. Iran. ${ }^{3}$ Department of Pathology, Hospital Universitario Central de Asturias, Oviedo, \\ 33006, Spain
}

\begin{abstract}
More than a century neurons with hyperstaining and compacted feature, dark neurons, have remained as a controversy in clinical and experimental neuropathology studies. Strictly speaking dark neuron formation is characterized with a series of morphological features and time-course stainability, but what made them quite bizarre is their non- enzymatic fashion of formation and reversibility. These enigmatic features led to emerging a new theory based on metastability of a gel- like structure filling the (neuro) cytoplasm. Although based on the gel- gel theory some mysterious aspects of dark neurons could be explained, role of receptors and cytoskeleton were not included. In practice, the term dark neuron is often known as the dead or degenerating neuron while in contrast more than 90-99 percent of dark neurons recover after a short period of time. The aim of this review is to give a clear explanation on the morphological features of dark neuron and also provide a theoretical framework in order to bridge between receptor- cytoskeleton and dark neuron behavior.
\end{abstract}

Key Words: Cytoskeleton, dark neurons, gel, neurodegeneration, receptor.

Revised: 1 October 2019, Accepted: 19 October 2019.

Corresponding Author: Arman Behrad, MD, Anatomy Department, Medicine Faculty, North Khorasan University of Medical Sciences, Bojnurd, Iran, Tel.: +989389455310, E-mail: Arman.behi@gmail.com

ISSN:2536-9172, December 2019, Vol. 3, No. 2

\section{INTRODUCTION}

Hyperstained dark shrunken neurons is a common finding in clinical and experimental neuropathology. The occurrence of dark stained neurons (DNs) has been a matter of debate among researchers. Since Flesch reported on neurons with hyperstaining features, a vast number of studies have reported such neurons which also known as hyperstained, compact, or dark neurons ${ }^{[1]}$. Maybe the first comprehensive study on DNs was carried out by Atillo A et al. 1983. His study showed that epileptic seizure is associated with DNs formation in the cortex of epileptic animals. According to Soderfeldt, DNs exhibit condensed dark staining with an increased rate of swollen mitochondria ${ }^{[2]}$. Subsequent studies in recent decades could show dark neuron formation in various neurologic disorders including epilepsy, pathometabolic condition, infection, aging, brain ischemia, and cholestasis ${ }^{[3-7]}$. Before the 1980s the prevailed notion only maintained extraneuronal causes of dark neuron formation (artefactual) ${ }^{[8-13]}$, but recent finding based on experimental studies have described four types of DNs; the artefactual, the neostriatal (the Huntington's type), the irreversible and reversible types ${ }^{[4]}$. Despite a vast number of reports on the presence of DNs in various experimental conditions, some researchers have questioned the essence of such a phenomenon and argue that DNs are nothing more than an artifact resulted from tissue processing ${ }^{[14-16]}$. Although this notion might be partially plausible in postmortem and non-preserved specimens, adequate perfusion fixation before tissue harvesting eliminates this type of DNs (artefactual type) ${ }^{[4,17]}$. Precisely DNs present neurons undergoing a series of morphological features like specific silver staining and hyperchromophilia, dark staining, and corkscrew appearance ${ }^{[18]}$. But what has made them quite bizarre and enigmatic are striking characteristics such as formation in non-enzymatic conditions and reversibility ${ }^{[19,20]}$. Studies have revealed that around $90-99 \%$ of dark neurons recover(reversible type) after a period of time, while in contrast, only a few proportions of dark neurons become dead neurons ${ }^{[21]}$. Electron microscopic and autoradiographic studies have demonstrated evidence confirming the low functional activity of dark shrunken neurons with intense protein synthesis due to the overexpression of amplified genes ${ }^{[22]}$. Nonetheless, a common feature of the all mentioned types is hyperstaining at the light microscopic level ${ }^{[8]}$. Because a majority of DNs with hyperchromophilia feature recover, the presence of hyperstained neurons should not be considered moribund or degenerating neurons. Therefore, their presence, without considering morphological features and time- course stainability, could be a potential source of unintentionally misleading in neuropathology studies. The origin and mechanism of dark neuron production remain unclear. Additionally, it remains to be clarified whether recovering neurons are still functional as normal neurons? 


\section{Identification and chronology of DNs occurrence:}

As mentioned before DNs have been reported in the various experimental conditions, pathometabolic, and even healthy normal animals ${ }^{[3-6,23]}$. So far no specific immunocytochemical method has been developed for DNs identification. Stainability of DNs with cationic or anionic dyes and argyrophilic are still used as the choice methods in light microscopic (LM) studies. Cationic dyes such as Mayer's hematoxylin and toluidine blue, anionic dyes such as acid fuchsin and eosin ${ }^{[24]}$, fluoro-jade,aminocupric-silver method of de Olmos ${ }^{[25,26]}$, physical and chemical - development - silver method of Gallyas et $a{ }^{[27,28]}$ have been used to demonstrate basophilia, acidophilia and argyrophilic of DNs during their phases of formation (Figure1-4). Chronologically DNs are classified into fresh, recovering, recovered, moribund, and dead ${ }^{[24-28]}$. Freshly - produced DNs(less than four hours from their formation) exhibit intense basophilia and argyrophilic, while DNs enter recovering phase gradually lose the acquired argyrphilia and basophilia and their normal basophilia reappeared ${ }^{[24]}$. The presence of small dark brown dots in cytoplasm of DNs or nuclear margin and nucleoli stainability with cationic dyes are considered recovering DNs (Figure 3-4). Moribund or dying DNs lose gradually their acquired and normal basophilia within few hours. In fact, a shift from mild acidophilia which reaches a strong acidophilia becomes observable in dying $\mathrm{DNs}^{[24]}$. In other words, DNs with survival time than 1 hour demonstrate basophilia, less than 48 hours exhibit good argyrophilia (Fig 3), while from 4 hours onward acidophilia gradually becomes more intense. Behind the dark appearance, DNs undergo a series of ultrastructural changes ${ }^{[24]}$. Based on transmission electron microscopy(TEM)fresh dark neurons(with survival time less than 1 hour)are characterized by dramatic volume reduction and increased electrondensity ${ }^{[4]}$. During compaction, a large volume of water passes through an apparently intact cell membrane and taken up by astrocytes. The next phase, sustained reaction, is characterized by more ultrastructural changes ( 1 hour after insult till 48 after survival time) including Golgi dilation, endoplasmic reticulum contraction, intracellular compaction, and finally chromatin clumping ${ }^{[20,21,24,29]}$. These ultrastructural changes (Fig 5-6) are accompanied by further three-dimensional neuronal shrinkage and compaction in the axon ${ }^{[29]}$.

To sum up dark neurons undergo a transient change in their electrical properties which is associated with reversible compaction. Different staining properties in dark neurons are probably suggestive of the conformational changes in proteins. An experimental study has yield evidence in favor of such an assumption ${ }^{[30,31]}$, but the mechanism of compaction or the trigger point for such cellular even has not been understood.

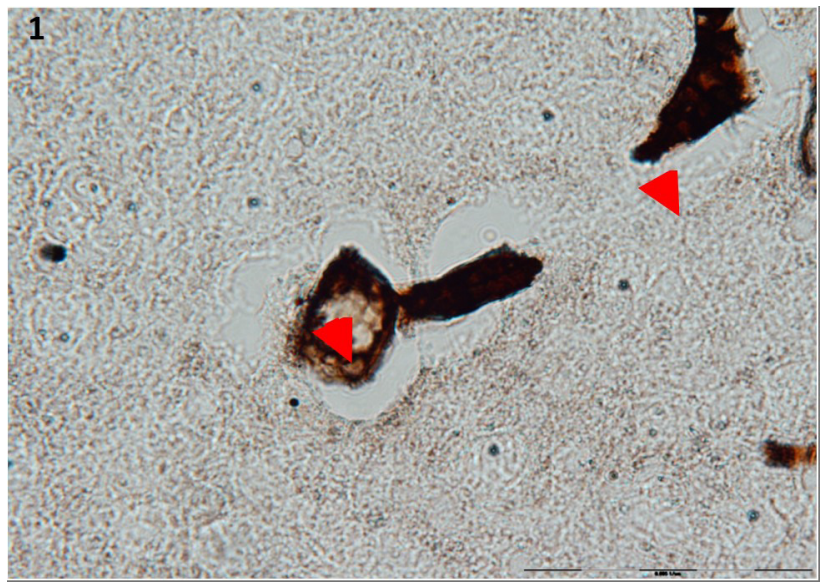

Fig 1: A degenerating dark neuron, detached from surrounding (red arrow heads). Degenerating dark neurons or moribund lose their argyrophilia gradually.

$\mathrm{X} 100$

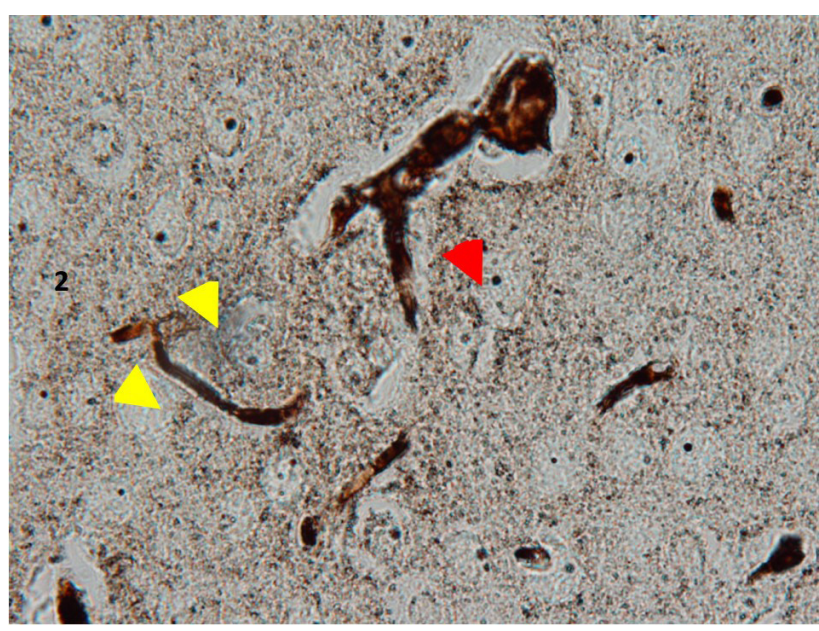

Fig 2: A dark neuron with dark brown stained appearance (red arrow head), in lower left corner a stained axon surrounded by healthy neurons (yellow arrowheads) are seen.

$\mathrm{X} 100$

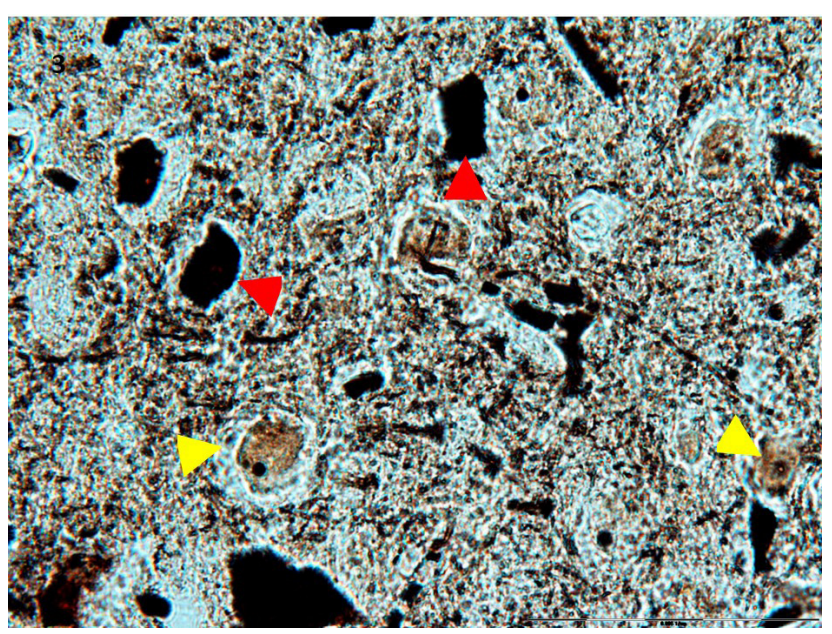

Fig 3: numerous deeply stained dark neurons with sharp contour (fresh dark neurons) are seen (red arrowheads), also scattered recovering dark neuron with amber like color cytoplasm (yellow arrowheads) are easily discernible. 


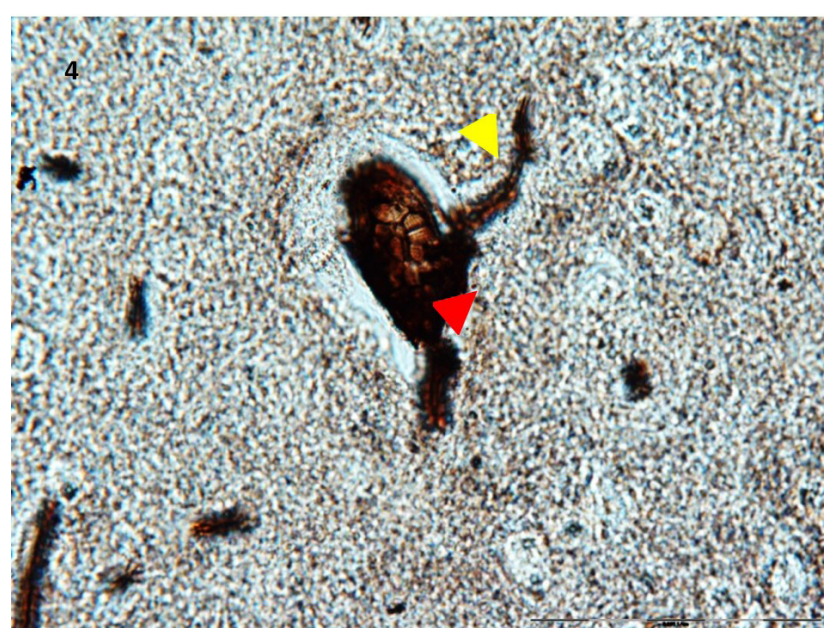

Fig 4: A typical dark neuron with Thread-like structure in its cytoplasm. Interestingly a synaptic neuronal process juxtaposed to the perikariyon showed darkening processes.

$\mathrm{X} 100$

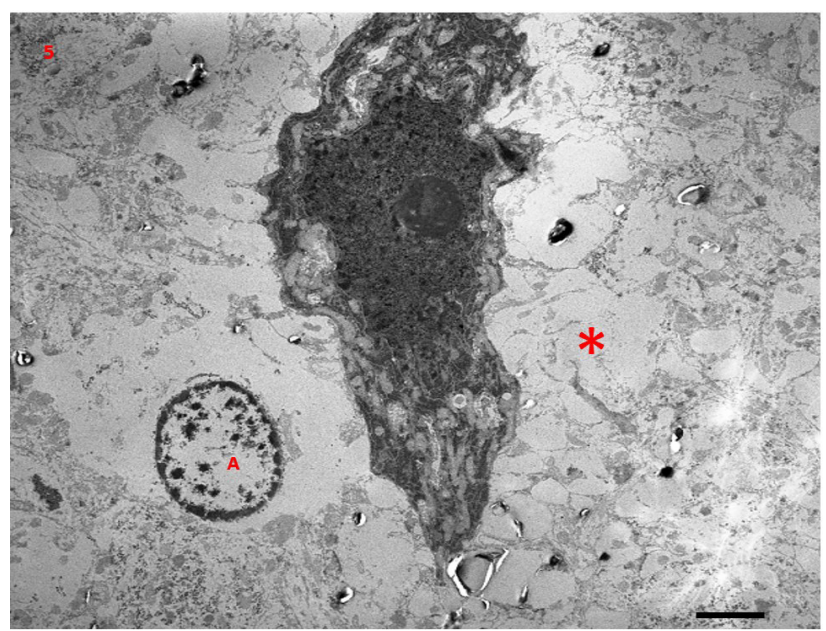

Fig 5: TEM photographs reveal morphological features of dark neurons. A dead dark neuron. Typically, a dark neuron is characterized with preserved cell and nucleus membranes, high electrondensity, dispersed chromatin pattern, irregular cell contour. Extensive vacuolization surrounding the neurons is evident indicating the reaction of astrocytes (A) during volume reduction. Scale bar 44 .

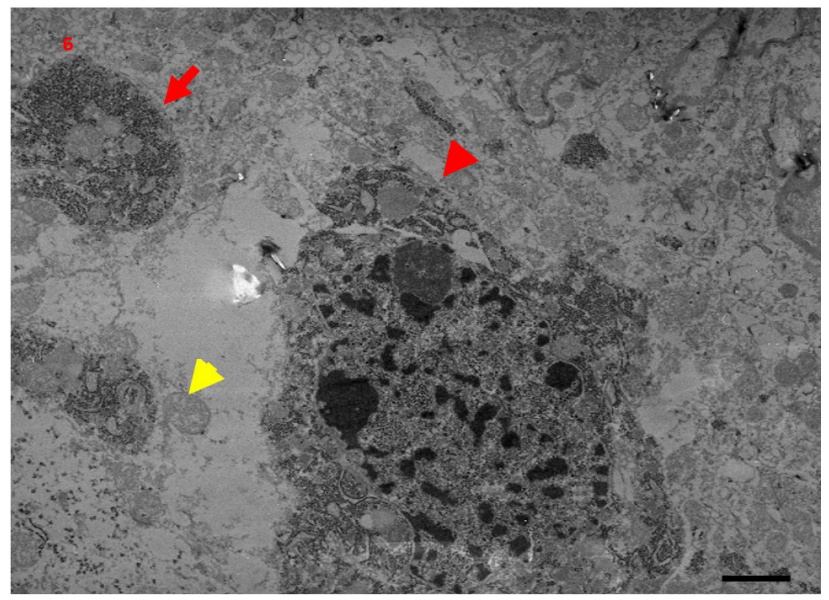

Fig 6: TEM photographs reveal morphological features of dark neurons. A dark neuron with compacted dark appearance. Chromatin pattern shows clumping and dispersion. Vacuolization surrounding the dark neuron and degenerating mitochondria (yellow arrowhead) are seen. In upper left a marked debris of degenerated neuron is noticeable (red arrow). Scale bar $2 \mu \mathrm{m}$.

\section{Reversibility mechanism}

A majority proportion of dark neurons enter the recovery phase so that more than $90-99 \%$ of DNs begin a spontaneous reverse scenario which is characterized by mitochondrion sized dot in the soma and subsequently in the dendrite ${ }^{[4,21,27]}$. Ultra structurally recovering neurons can be outlined with endoplasmic dilation and delivery of membranous whorls bodies to astrocytes ${ }^{[20,29]}$. About $1-10 \%$ of DNs may enter an irreversible process in which cellular changes lead to fragmentation of chromatin, vacuolization, and finally, become dead neurons (vide infra) ${ }^{[32]}$. Despite the vast number of studies the underlying mechanism(s) of dark neurons has not been fully revealed. At present the prevailed hypothesis of the mechanism of DNs formation gives much more credence to the gel-to-gel transition phase in the cytoplasm(hyaloplasm) of neurons undergoing compaction $^{[20]}$. According to this theory, an enigmatic structure with metastable form fills the spaces between organelles. This structure stores noncovalent energy that can be released after exposure to various noxae and so a chain of chemical reactions like polymerization would begin and spread (domino likes effect) gel-gel hypothesis ${ }^{[33]}$. By polymerization of intraneuronal gel morphologic characteristics of DNs such as volume reduction, shrinkage and electrondensity would be explainable, but the link between extracellular - intracellular milieu and propagation of this phenomenon remains obscure ${ }^{[34,35]}$. Despite the relative compatibility of the gel-to-gel phase theory in explanation of the DNs phenomenon, it ignores the role of receptors and cytoskeleton in DNs formation. For instance, the study has documented evidence that suggests the role of the cytoskeleton in dark neurons formation $^{[30,31]}$. Studies emphasize the role of glutamate receptors in dark neuron formation ${ }^{[23,36]}$. On the other hand, there are reports on the occurrence of such neurons in normal healthy conditions, which indicate the developmental role of dark neurons formation in the CNS. Tsubouchi et al reported DNs presence during post-natal life. They also found that DNs exhibit different affinity to lectin in comparison with those of other neurons. He concluded that DNs are presumably exhausted neurons after a period of activity $^{[37]}$. Besides Vohra et al could show the occurrence of DNs in the aging cerebellum ${ }^{[6]}$. Our previous studies showed diabetes mellitus (uncontrolled hyperglycemia) increases DNs formation in the hippocampus. Interestingly a few scattered DNs were also seen in the hippocampus of the normal control groups ${ }^{[4]}$. Now a question may be raised that; should the significantly increased number of dark neurons in a pathometabolic condition such diabetes is treated as acceleration in degeneration or more vulnerability? Although the term "dark neuron" has been used synonymously in pieces of literature, it needs to take caution in the application of the term dark neurons, for instance, the term dark neurons in health conditions such as postnatal development should be interpreted as naturally occurring dark neuron (NODN), while dark neurons occurring in aging process should be considered a way of neuronal death. 


\section{Fate of DNs}

The mode of death of DNs has not been fully defined and data on the fate of DNs are controversial. According to TEM findings, the mode of death in DNs could not be categorized into necrotic or apoptotic death ${ }^{[3,38,39]}$, but the similarities between apoptotic death and the mode of death in dead DNs could be suggestive of a possible common compaction and degradation process ${ }^{[4,40]}$.

\section{A common target of different insults governs plasticity-like nature of DNs}

DNs undergo a series of morphological characteristics including volume reduction and shrinkage. The nature of such a phenomenon would presumably depend on the rapid reaction of cytoskeletal elements ${ }^{[30,31]}$. Therefore it would be arguable to be assumed (i) a variety of different noxae, share in a common target (ii) that can transmit microphysical or traumatic stress (iii) to the cytoskeleton bounded to the cell membrane. A question may arise that how could the occurrence of DNs in healthy conditions $^{[4,}{ }^{37]}$ or during postnatal development ${ }^{[6]}$ be explained? It might be argued that such neurons are probably apoptotic neurons, but the point is that less than $10 \%$ of dark neurons enter the "dead" phase ${ }^{[3,27-29,38-40]}$. Additionally, the presence of recovering dark neurons in the case of animal studies underlines the vulnerability of the neuro-glial defense protective mechanism in harmful paradigms ${ }^{[27]}$. Therefore, it would be arguable to assume that when a dark neuron regains its normal morphology, exhibiting a plastic-like behavior. In other words, the comparison between the dynamic nature of DNs and the behavior of neurons during plasticity likely reflects a common developmental mechanism. Reversibility of DNs phenomenon in particular shrinkage and surface reduction can be analogized to the plasticity. Essentially plasticity is referred to the peerless ability of the brain (neuron) to change and reorganize in response to changes in the environment. This unique ability of neurons promotes their viability and subsequently survival of the organism ${ }^{[41]}$. The most known examples of neuronal plasticity are new synapses formation, dendrite spines sprouting, retraction and simplification of dendrites, and reduction of dendritic spines in stressful conditions. Studies have revealed that endogenous or exogenous stressors are associated with a decrease in the surface and dendritic spine of the neurons ${ }^{[42]}$. Although such reductive responses are mediated by various signaling pathways and hormones like cortisol (in animals), the rationale behind these reactions has been less noticed. Simplification of the dendritic tree and spine reduction could be considered a protective mechanism by which a neuron protects itself against insults (neural Darwinism) ${ }^{[43]}$. An intermediate structure likely mediates as an external sensor. Such a role could be attributed to the cell surface coat. The outer surface of a most mammalian cell is covered by glycoprotein composed of oligosaccharides and proteins. Glycoprotein plays a pivotal role in various cell behaviors including cell to cell interaction, stabilizing, and protective functions ${ }^{[44]}$. The protective function of glycoprotein is speculated to be vital to the central nervous system (CNS) due to its limited regeneration capacity ${ }^{[45]}$. It has been proposed that glycoprotein reduces the sensitivity of neurons to glutamate excitotoxicity and damage ${ }^{[46]}$. A study by Kherani et al demonstrated that glutamate release or receptor activation are more likely mechanisms of dark neuron production ${ }^{[36]}$. Semiempirical molecular orbital method- based study suggests that different vibrational states of glutamate receptors determine the excitation or relaxation of a neuron ${ }^{[47]}$. Accordingly, the outer coat of neurons (say acid sialic) is of a vibration state that would inhibit actin polymerization. Changes in the vibrational state of glycocalyx would determine the transient increase in intracellular pressure, leading to volume reduction and vice versa. Subsequently, any transient deviation of glycocalyx vibrational state could affect cytoskeletal meshwork, nucleus membrane-tethered to the cytoskeleton, and actin $^{[48]}$. These chains of reactions mediate actin polymerization, contraction of the cytoskeleton, and eventually transient increase in intraneuronal pressure which in turn leading to water loss and cell shrinkage. A unidirectional outflux of water and probably ions will affect some organelles like mitochondria which are sensitive to ion gradients ${ }^{[49]}$. In tandem with these changes, due to the connection between cytoskeletal elements and the nucleus membrane, increased intracellular pressure would affect the nucleus and chromatin, leading to chromatin clumping and margination. The reversibility of these phenomena will depend on returning of glycocalyx vibrational state to the specific regional defined range and actin depolymerization. Ultrastructural and light microscopic findings confirm this notion that the cytoskeleton complex is contributed to DNs formation. Thread-like structures similar to neurofibrillary tangle (NFT) may confirm cytoskeleton involvement in DNs. Sever insult may disrupt glycocalyxcytoskeleton complex, inducing neurofibrillary tangle that is the hallmark of neurodegeneration ${ }^{[50]}$. It seems the glycocalyx-cytoskeleton complex is suited to act as a mechanotransducer that could stir cytoskeleton reaction ${ }^{[48]}$. Therefore, it would reasonable to assume any change including physical or chemical in extracellular milieu may affect the normally defined limit of glycocalyxcytoskeleton and leads to darkening.

\section{Propagation of dark neuron phenomenon}

Another exceptional feature of the $\mathrm{DN}$ formation phenomenon is self-spreading through the dendrites and soma ${ }^{[33-35]}$. Although it has been remarked this phenomenon would restrict to dendrites and soma, there is good evidence demonstrating the axonal involvement ${ }^{[4]}$. According to the previous reports on the dark neuron phenomenon, it has been stated that it could propagate to adjacent neurons through the dendrite connections ${ }^{[3,19-21,28,29,33-35]}$. A study by Hajnal et al demonstrated that amygdaloid kainite lesion causes dark neuron formation in the anatomical related area possibly through the neuronal projection ${ }^{[51]}$. The connection between two proximity neurons occurs in the synaptic 
region. Ergo DNs formation is presumably related to the Spatio-temporal arrangement of neuronal connections. Direct connections of juxtaposed neurons seem to be a sine qua non to spreading the DN phenomenon. This type of connection may be responsible for fast, synchronous, and coherent brain activity during conscious processing or $\mathrm{EEG}^{[52]}$. Recent studies have yield evidence indicating the presence of physical connection in the synaptic region. Presynaptic and postsynaptic membranes of dendrites are linked by $\beta$ neurexin and neuroligin. The $\beta$ neurexinneuroligin adhesion can influence the cytoskeletons of the two adjacent neurons ${ }^{[53]}$. The intracellular domains of these adhesive proteins establish a connection with the presynaptic domain zone (PDZ) and postsynaptic density (PSD). Cell adhesion molecules neurexin (presynaptic) and neuroligin (presynaptic) connect (or indirectly by linking synaptic proteins) to the cytoskeleton through the actinbinding protein 4.1 or the microtubule-binding protein CRIPT $^{[54]}$. This sort of connection provides fast intracellular connection, resulting in actin polymerization of the two closely apposed neurons. We speculate, concerning the role $\beta$ neurexin-neuroligin adhesion, polymerization of actin, and transition phase from one neuron can spread and affect neighboring neurons. To conclude, dark neuron formation is a reversible, dynamic, and self-propagate response to the transient alteration in the extracellular physic-chemical milieu. Considering the fact that the majority of dark neurons regain their normal morphology, dealing more with just their formation in a variety of conditions would not be helpful. Given less than 10 percent of dark neurons enter an irreversible degenerative process; it would be worthwhile to consider that dark neurons occurrence potentially heralds neuronal loss. The relation between their occurrence and any functional disturbance or cognitive deficit needs more investigation in the future.

\section{CONFLICT OF INTEREST}

There are no conflicts of interest.

\section{REFERENCES}

1. Johnson JEJAn. The occurrence of dark neurons in the normal and deafferentated lateral vestibular nucleus in the rat. 1975;31(2):117-27.

2. Atillo A, Soderfeldt B, Kalimo H, Olsson Y, Siesjo BK. Pathogenesis of brain lesions caused by experimental epilepsy. Light- and electronmicroscopic changes in the rat hippocampus following bicuculline-induced status epilepticus. Acta Neuropathol. 1983;59(1):11-24.

3. Gallyas F, Kiglics V, Baracskay P, Juhasz G, Czurko A. The mode of death of epilepsy-induced "dark" neurons is neither necrosis nor apoptosis: an electron-microscopic study. Brain Res. 2008;1239:207-15.

4. Ahmadpour SH, Haghir H. Diabetes mellitus type 1 induces dark neuron formation in the dentate gyrus: a study by Gallyas' method and transmission electron microscopy. Rom J Morphol Embryol. 2011;52(2):575-9.

5. Auer RN, Kalimo H, Olsson Y, Siesjo BK. The temporal evolution of hypoglycemic brain damage. I. Light- and electron-microscopic findings in the rat cerebral cortex. Acta Neuropathol. 1985;67(12):13-24

6. Vohra BP, James TJ, Sharma SP, Kansal VK, Chudhary A, Gupta SK. Dark neurons in the ageing cerebellum: their mode of formation and effect of Maharishi Amrit Kalash. Biogerontology. 2002;3(6):347-54.

7. Bon LI, Maksimovich N, Zimatkin SM. Effects of experemental cerebral ishemia on metabolic characteristics of parietal cortex neurons. 2018.

8. Cammermeyer J. The post-mortem origin and mechanism of neuronal hyperchromatosis and nuclear pyknosis. Exp Neurol. 1960;2:379-405.

9. Cammermeyer J. The importance of avoiding "dark" neurons in experimental neuropathology. Acta Neuropathologica. 1961;1(3):245-70.

10. Cammermeyer J. An evaluation of the significance of the "dark" neuron. . Ergebnisse der Anatomie und Entwicklungsgeschichte. 1962;36:1-61.

11. Cammermeyer J. Nonspecific changes of central nervous system in normal and experimental material. In: $\mathrm{GH}$ B, editor. The structure and function of nervous tissue, 6. New York: Academic Press; 1972. p. 131-251.

12. Cammermeyer J. Is the solitary neuron a manifestation of postmortem trauma to the brain inadequately fixed by perfusion? Histochemistry. 1978;56:97-115.

13. Scharrer E. On dark and light cells in the brain and in the liver. Anat Rec. 1938;72:53-65.

14. Capo I, Lalosevic D. [Interpretation of dark neurons in experimental model of ischemia, neurointoxication and brain infection]. Med Pregl. 2011;64(1-2):101-6. 
15. Garman RH. The return of the dark neuron. A histological artifact complicating contemporary neurotoxicologic evaluation. Neurotoxicology. 2006;27(6):1126.

16. Jortner BS. The return of the dark neuron. A histological artifact complicating contemporary neurotoxicologic evaluation. Neurotoxicology. 2006;27(4):628-34.

17. EM Løberg AT. Distinction between artefactually shrunken and truly degenerated 'dark' neurons by in situ fixation with microwave irradiation. Neuropathol Appl Neurobiol. 1993;19(4):359-63.

18. Zsombok A, Tóth Z, Gallyas FJJonm. Basophilia, acidophilia and argyrophilia of "dark"(compacted) neurons during their formation, recovery or death in an otherwise undamaged environment. 2005;142(1):145-52.

19. Gallyas F PJ. Whole-Cell Phase Transition in Neurons and its Possible Role in Apoptotic Cell Death. Phase Transitions in Cell Biology. 2008:6371.

20. Kellermayer R, Zsombok A, Auer T, Gallyas F. Electrically induced gel-to-gel phase-transition in neurons. Cell Biol Int. 2006;30(2):175-82.

21. Csordas A, Mazlo M, Gallyas F. Recovery versus death of "dark" (compacted) neurons in non-impaired parenchymal environment: light and electron microscopic observations. Acta Neuropathol. 2003;106(1):37-49.

22. Kalimullina LJM. " Dark" and" light" cells. 2002;122(4):75

23. Ahmadpour S, Foghi K, Behrad AJJoMS. Chronic exposure to ketamine induces neuronal lose and glial reaction in CA4 region of hippocampus. 2016;33(02):103-7.

24. Zsombok A, Toth Z, Gallyas F. Basophilia, acidophilia and argyrophilia of "dark" (compacted) neurons during their formation, recovery or death in an otherwise undamaged environment. J Neurosci Methods. 2005;142(1):145-52.

25. Schmued LC, Hopkins KJ. Fluoro-Jade B: a high affinity fluorescent marker for the localization of neuronal degeneration. Brain Res. 2000;874(2):123-30.

26. Carlsen J, De Olmos JS. A modified cupric-silver technique for the impregnation of degenerating neurons and their processes. Brain Res. $1981 ; 208(2): 426-31$

27. Gallyas F, Hsu M, Buzsaki G. Four modified silver methods for thick sections of formaldehyde-fixed mammalian central nervous tissue: 'dark' neurons, perikarya of all neurons, microglial cells and capillaries. J Neurosci Methods. 1993;50(2):15964.

28. Gallyas F, Zoltay G, Dames W. Formation of "dark" (argyrophilic) neurons of various origin proceeds with a common mechanism of biophysical nature (a novel hypothesis). Acta Neuropathol. 1992;83(5):504-9.

29. Gallyas F, Farkas O, Mazlo M. Gel-to-gel phase transition may occur in mammalian cells: Mechanism of formation of "dark" (compacted) neurons. Biol Cell. 2004;96(4):313-24.

30. Inoue $\mathrm{K}$, Fujimoto I, Fukuda A, Nishino $\mathrm{H}$. Microtubule-associated protein 2 expressing COS7 cells are resistant to argyrophilia under oxygen- and glucose-free condition. Exp Neurol. 1996;137(2):350-6.

31. Ishida K, Ungusparkorn C, Hida H, Aihara N, Ida K, Nishino H. Argyrophilic dark neurons distribute with a different pattern in the brain after over hours treadmill running and swimming in the rat. Neurosci Lett. 1999;277(3):149-52.

32. Toth Z, Seress L, Toth P, Ribak CE, Gallyas F. A common morphological response of astrocytes to various injuries: "dark" astrocytes. A light and electron microscopic analysis. J Hirnforsch. 1997;38(2):173-86.

33. Gallyas F. A cytoplasmic gel network capable of mediating the conversion of chemical energy to mechanical work in diverse cell processes: a speculation. Acta Biol Hung. 2010;61(4):367-79.

34. Gallyas F, Pal J, Bukovics P. Supravital microwave experiments support that the formation of "dark" neurons is propelled by phase transition in an intracellular gel system. Brain Res. 2009;1270:152-6.

35. Gallyas F, Zoltay G, Horvath Z, David K, Kelenyi L. An immediate morphopathologic response of neurons to electroshock; a reliable model for producing "dark" neurons in experimental neuropathology. Neurobiology (Bp). 1993;1(2):133-46. 
36. Kherani ZS, Auer RN. Pharmacologic analysis of the mechanism of dark neuron production in cerebral cortex. Acta Neuropathol. 2008;116(4):447-52.

37. Tsubouchi M, Tsubouchi Y, Hitomi S, Ohtsuka A, Murakami T. Perineuronal sulfated proteoglycans, cell surface glycoproteins and dark neurons in the cingulate cortex of newborn and adult rats. Acta Med Okayama. 1996;50(6):313-7.

38. Kovesdi E, Pal J, Gallyas F. The fate of "dark" neurons produced by transient focal cerebral ischemia in a non-necrotic and non-excitotoxic environment: neurobiological aspects. Brain Res. 2007; 1147:272-83.

39. Gallyas F. Novel cell-biological ideas deducible from morphological observations on "dark" neurons revisited. Ideggyogy Sz. 2007;60(56):212-22.

40. SH Ahmadpour YSaHH. Streptozotocin-induced Hyperglycemia Produces Dark Neuron in CA3 Region of Hippocampus in Rats. Asian Journal of Medical Sciences. 2010;2(1):11-5.

41. Marder E, Prinz AA. Current compensation in neuronal homeostasis. Neuron. 2003;37(1):2-4.

42. McEwen BS, Nasca C, Gray JD. Stress Effects on Neuronal Structure: Hippocampus, Amygdala and Prefrontal Cortex. Neuropsychopharmacology. 2015.

43. Dale Purves LEWaDRR. Is neural development darwinian? Trends Neurosci. 1996;19:460-4.

44. Yoo SW, Motari MG, Susuki K, Prendergast J, Mountney A, Hurtado A, et al. Sialylation regulates brain structure and function. FASEB J. 2015.

45. Horner PJ, Gage FH. Regenerating the damaged central nervous system. Nature. 2000;407(6807):963-70.
46. Mehta NR, Nguyen T, Bullen JW, Jr., Griffin JW, Schnaar RL. Myelin-associated glycoprotein (MAG) protects neurons from acute toxicity using a ganglioside-dependent mechanism. ACS Chem Neurosci. 2010;1(3):215-22.

47. Kubo M, Odai K, Sugimoto T, Ito E. Quantum chemical study of agonist-receptor vibrational interactions for activation of the glutamate receptor. J Biochem. 2001;129(6):869-74.

48. Ramdas NM, Shivashankar GV. Cytoskeletal control of nuclear morphology and chromatin organization. J Mol Biol. 2015;427(3):695-706.

49. Raimundo N. Mitochondrial pathology: stress signals from the energy factory. Trends Mol Med. 2014;20(5):282-92.

50. Brandt R, Hundelt M, Shahani N. Tau alteration and neuronal degeneration in tauopathies: mechanisms and models. Biochim Biophys Acta. 2005;1739(2-3):331-54

51. Hajnal A, Lenard L, Czurko A, Sandor P, Karadi Z. Distribution and time course of appearance of "dark" neurons and EEG activity after amygdaloid kainate lesion. Brain Res Bull. 1997;43(2):235-43.

52. Cunningham MO, Roopun A, Schofield IS, Whittaker RG, Duncan R, Russell A, et al. Glissandi: transient fast electrocorticographic oscillations of steadily increasing frequency, explained by temporally increasing gap junction conductance. Epilepsia. 2012;53(7):1205-14.

53. Biederer T, Stagi M. Signaling by synaptogenic molecules. Curr Opin Neurobiol. 2008;18(3):2619.

54. Hata Y, Butz S, Sudhof TC. CASK: a novel dlg/ PSD95 homolog with an N-terminal calmodulindependent protein kinase domain identified by interaction with neurexins. $J$ Neurosci. 1996;16(8):2488-94 